\title{
Riscos e ocorrências de eventos adversos na percepção de enfermeiros assistenciais*
}

\author{
Risks and occurrences of adverse events in the perception of health care nurses
}

Robson Tostes Amaral ${ }^{1}$, Ana Lúcia Queiroz Bezerra ${ }^{1}$, Cristiane Chagas Teixeira ${ }^{1}$, Thatianny Tanferri de Brito Paranaguá ${ }^{2}$, Thaisa Cristina Afonso ${ }^{1}$, Adrielle Cristina Silva Souza ${ }^{1}$

Objetivo: analisar os riscos e ocorrências de eventos adversos em pacientes hospitalizados na perspectiva de enfermeiros. Métodos: estudo de corte transversal, desenvolvido com 41 enfermeiros assistenciais. Utilizouse instrumento autoaplicável intitulado Eventos Adversos Associados às Práticas de Enfermagem validado em Portugal e adaptado à realidade brasileira. Resultados: a quantidade de vínculos empregatícios $(\mathrm{p}=0,019) \mathrm{e} a$ carga horária semanal $(\mathrm{p}=0,002)$ se mostraram potencializadores de falhas nos cuidados assistenciais. Houve correlação positiva entre lesões por pressão e quedas $(\mathrm{p}<0,001)$, erros de medicamentos e lesões por pressão $(\mathrm{p}=0,004)$ e infecções relacionadas à assistência à saúde e erros de medicamentos $(\mathrm{p}=0,006)$. Conclusão: $\mathrm{a}$ análise evidenciou que a ocorrência de eventos adversos nos cuidados assistenciais como lesões por pressão e infecções relacionadas à assistência à saúde foram as mais frequentes na percepção de enfermeiros. Dos domínios explorados, erros de medicamentos apontou entre os resultados com menor incidência.

Descritores: Segurança do Paciente; Cuidados de Enfermagem; Dano ao Paciente.

Objective: to analyze the risks and occurrences of adverse events in hospitalized patients from the perspective of nurses. Methods: cross-sectional study, developed with 41 nurses. We used a self-administered instrument titled Adverse Events Associated with Nursing Practices validated in Portugal and adapted to the Brazilian reality. Results: the amount of employment bonds $(p=0.019)$ and weekly workload $(p=0.002)$ foster failures in care assistance. There was a positive correlation between pressure and falls injuries $(p<0.001)$, medication errors and pressure injuries $(\mathrm{p}=0.004)$ and infections related to health care and medication errors $(\mathrm{p}=0.006)$. Conclusion: the analysis showed that the occurrence of adverse events in care such as pressure injuries and infections related to health care were the most frequent in the perception of nurses. Drug errors had the lowest incidence among the results of the explored domains.

Descriptors: Patient Safety; Nursing Care; Patient Harm.

\footnotetext{
*Extraído da Dissertação "Práticas assistenciais e ocorrência de eventos adversos: percepção dos enfermeiros", Universidade Federal de Goiás, 2018.

${ }^{1}$ Universidade Federal de Goiás. Goiânia, GO, Brasil.

${ }^{2}$ Universidade de Brasília. Brasília, DF, Brasil.
} 


\section{Introdução}

Debates a respeito da qualidade da assistência e da segurança do paciente tem sido tendência global em decorrência da existência de riscos e ocorrências de eventos adversos em ambientes hospitalares. Garantir a segurança dos pacientes hospitalizados ainda é um grande desafio para os sistemas de saúde e profissionais de enfermagem ${ }^{(1)}$.

Estudos realizados em hospitais portugueses e canadenses mostram a incidência de $12,5 \%$ a $15,3 \%$ de eventos adversos nas práticas assistenciais de saúde, sendo frequentes as lesões por pressão, quedas, erros na administração de medicamentos, infecções hospitalares e procedimentos cirúrgicos ${ }^{(1-2)}$. Dentre os fatores que contribuem para a ocorrência dos eventos adversos, destacam-se o déficit de pessoal, sobrecarga de trabalho, conflitos entre os profissionais, falta de liderança e supervisão inadequada ${ }^{(3)}$.

Uma análise realizada em sete hospitais no Brasil identificou que todos possuíam sistema de notificação de eventos adversos, 71,4\% dispunham de Comissão de Gerenciamento de Riscos, 80,0\% realizavam discussões dos eventos, havendo consenso para mudanças favoráveis à segurança do paciente. Todavia, o receio dos colaboradores por punição devido às suas falhas e a subnotificação dos eventos ainda estavam presentes no ambiente de trabalho ${ }^{(4)}$, o que revela que ainda é necessário avançar no fortalecimento da cultura de segurança.

Na saúde, a enfermagem configura-se como a maior força de trabalho, incentivadora de estratégias de segurança do paciente e prevenção de eventos adversos, com articulação e cooperação entre as instituições de saúde e a educação de profissionais(5). Entretanto, o profissional de saúde precisa reconhecer, perceber e discutir sobre as situações de risco para colaborar com o adequado gerenciamento do serviço e da assistência, a prevenção de falhas e o estabelecimento da cultura de segurança na organização hospi$\operatorname{talar}^{(3)}$.

No cenário de avaliação de serviços, conside- rando os avanços no setor saúde e o âmbito da enfermagem quanto ao seguimento das políticas de segurança do paciente, levanta-se a seguinte questão: qual a perspectiva de enfermeiros de um hospital de ensino quanto aos riscos e ocorrências de eventos adversos em pacientes hospitalizados, frente à organização e à gestão do serviço de saúde?

A análise das falhas que ocorrem na assistência pode auxiliar na identificação de processos organizacionais que precisam ser melhorados a fim de fortalecer a cultura de segurança, especialmente, quanto à omissão de notificação de eventos adversos pelo sentimento de culpa, estresse emocional e medo de punições frente aos preceitos éticos a que estão expostos, bem como na mitigação de riscos e ocorrências de eventos adversos nas práticas assistenciais ${ }^{(5)}$. Considerando a necessidade de desenvolver políticas organizacionais que considerem todos os profissionais como sujeitos ativos no processo de gerenciamento de riscos, teve-se como objetivo analisar os riscos e ocorrências de eventos adversos em pacientes hospitalizados na perspectiva de enfermeiros.

\section{Métodos}

Trata-se de um estudo de corte transversal desenvolvido nas unidades de clínica médica, cirúrgica e terapia renal substitutiva de um hospital de ensino estadual da região Centro-Oeste do Brasil. Acreditado no nível dois pela Organização Nacional de Acreditação dispõe de Núcleo de Segurança do Paciente estruturado e atuante desde 2013.

Considerando o foco do estudo, os critérios de inclusão da população foram: trabalhar na assistência direta ao paciente e tempo de trabalho, na assistência, por período igual ou maior que seis meses, na instituição do estudo. A população foi composta por 45 enfermeiros assistenciais, obtendo taxa de resposta de 41 $(91,1 \%)$ participantes. Quatro enfermeiros não participaram da pesquisa pois estavam de licença durante o período de coleta de dados.

Os dados foram coletados no período de janeiro 
a maio de 2017, utilizando dois instrumentos. 0 primeiro, construído para fins desse estudo, investigou a caracterização sociodemográfica e profissional dos participantes como sexo, idade, tempo de formação profissional, tempo de atuação profissional, quantidade de vínculos empregatícios, carga horária semanal, curso na área de segurança do paciente e curso de qualificação.

O segundo instrumento consistiu na escala Eventos Adversos Associados às Práticas de Enfermagem, construída e validada em Portugal ${ }^{(6-7)}$ e adaptado para o Brasil. Trata-se de uma escala de análise de riscos e ocorrências de eventos adversos associados às práticas de enfermagem, autoaplicável, com domínios relacionados aos indicadores de resultado das práticas assistenciais, quais sejam: agravamento e complicações por déficit de vigilância e julgamento clínico inadequado, déficit de defesa e delegação inapropriada de um profissional menos preparado para realização de cuidados, quedas, lesões por pressão, erros de medicamentos e infecção relacionada à assistência à saúde, considerados como variáveis de desfecho.

Os itens foram respondidos em uma escala de tipo Likert de cinco pontos, correspondendo o escore (1) a Nunca, (2) a Raramente, (3) a Algumas vezes, (4) a Frequentemente e (5) a Sempre. As frequências do instrumento foram agrupadas em três itens: nunca e raramente, algumas vezes, frequentemente e sempre, para facilitar a visualização dos resultados encontrados.

Os dados coletados foram analisados nos programas Statistical Package for the Social Sciences versão 24.0 e Stata, versão 14.0. Inicialmente se verificou a normalidade das variáveis quantitativas, utilizando o teste de Kolmogorov-Smirnov. Para análise bivariada, utilizou-se os testes de correlação de Spearman para as variáveis quantitativas e o teste de Wilcoxon-Mann-Whitney ou Kruskal-Wallis para as qualitativas. A análise descritiva dos itens e domínios foi realizada com média, desvio padrão, mínimo, máximo e porcentagem. A análise da confiabilidade interna das subescalas foi feita pelo alfa de Cronbach padronizado, com confiabilidade interna $\geq 0,7$.

0 estudo foi aprovado pelo Comitê de Ética em Pesquisa do Hospital das Clínicas da Universidade Federal de Goiás e instituição coparticipante sob parecer no $1.876 .211 / 2016$. Todos os participantes assinaram o Termo de Compromisso Livre e Esclarecido.

\section{Resultados}

Entre os 41 enfermeiros, $31(75,6 \%)$ eram do sexo feminino, a média de idade foi de 33 anos, 19 (46,3\%) possuíam um vínculo empregatício e 22 $(53,7 \%)$ dois vínculos. A carga horária semanal apresentou média de 56,2 horas. 0 tempo de formado e atuação profissional foi entre cinco e 10 anos, respectivamente, para $23(56,1 \%)$ e $17(41,5 \%)$ profissionais. Nos últimos 12 meses, $26(63,4 \%)$ realizaram algum curso de capacitação em segurança do paciente. Quanto ao curso de qualificação, 19 (46,3\%) possuíam alguma especialização, incluindo um enfermeiro que cursou doutorado.

Na correlação entre as variáveis do perfil profissional e o risco e ocorrência de eventos adversos, observou-se associação positiva entre enfermeiros com mais de um vínculo empregatício $(\mathrm{p}=0,019)$ e maior carga horária semanal $(\mathrm{p}=0,002)$ com a delegação inapropriada de profissionais menos preparados para a assistência. A extensa carga horária semanal de trabalho aumentou o risco e ocorrência de eventos adversos com medicamentos $(\mathrm{p}=0,012)$. A participação do enfermeiro em curso de capacitação em segurança do paciente indicou redução do risco e ocorrência de eventos adversos relacionados a lesões por pressão $(\mathrm{p}=0,019)$.

Apesar de 58,5\% dos enfermeiros relatarem que nunca ou raramente ocorrem complicações do paciente por julgamento clínico inadequado, 46,3\% apontaram algumas vezes a ocorrência de eventos adversos relacionados a quedas e $14,6 \%$ afirmaram que as lesões por pressão são frequentes na instituição. Ainda que $80,5 \%$ dos enfermeiros tenham percebido que nunca ou raramente ocorre risco de complicações 
do paciente por delegação inapropriada de profissional menos preparado para a assistência, $4,9 \%$ dos enfermeiros confirmaram ser frequente a ocorrência de erros de medicamentos e apenas $26,8 \%$ negaram a ocorrência de infecções relacionada à assistência à saúde no processo assistencial. As demais análises de eventos adversos estão demonstradas na Tabela 1.

Observou-se que a percepção sobre o risco de quedas, lesões por pressão, erros de medicamentos e infecções relacionadas à assistência à saúde na ins- tituição foi maior que a percepção de ocorrência. Ao analisar os domínios, verificou-se que todas as práticas assistenciais percebidas pelos enfermeiros obtiveram média de 2,5 ou mais, exceto pelo domínio 'Déficit de defesa e delegação inapropriada'. 0 alfa de Cronbach apresentou variação dos domínios entre 0,566 a 0,778. 0 domínio que abordou o risco de agravamento e complicações do paciente por delegação inapropriada mostrou boa confiabilidade, $\operatorname{com} \alpha=0,77$. Os demais domínios obtiveram confiabilidade de consistência de baixa a moderada (Tabela 2).

Tabela 1 - Análise de riscos e ocorrências de eventos adversos na perspectiva de enfermeiros de um hospital de ensino $(n=41)$

\begin{tabular}{|c|c|c|c|c|c|c|}
\hline \multirow[t]{2}{*}{ Domínios } & $\begin{array}{c}\text { Nunca/ } \\
\text { Raramente }\end{array}$ & $\begin{array}{c}\text { Algumas } \\
\text { vezes }\end{array}$ & $\begin{array}{c}\text { Frequente- } \\
\text { mente/Sempre }\end{array}$ & \multirow[t]{2}{*}{ Média (DP*) } & \multirow[t]{2}{*}{ Mínimo } & \multirow[t]{2}{*}{ Máximo } \\
\hline & n (\%) & n (\%) & n (\%) & & & \\
\hline Déficit de vigilância & $17(41,5)$ & $19(46,3)$ & $5(12,2)$ & $2,7(0,8)$ & 1,0 & 4,0 \\
\hline Julgamento clínico inadequado & $23(58,5)$ & $15(36,6)$ & $2(4,9)$ & $2,4(0,7)$ & 1,0 & 4,0 \\
\hline Déficit de defesa & $27(65,9)$ & $11(26,8)$ & $3(7,3)$ & $2,3(0,8)$ & 1,0 & 4,0 \\
\hline Delegação inapropriada & $33(80,5)$ & $5(12,2)$ & $3(7,3)$ & $1,8(0,9)$ & 1,0 & 5,0 \\
\hline \multicolumn{7}{|l|}{ Quedas } \\
\hline Risco & $9(22,0)$ & $20(48,8)$ & $12(29,3)$ & $3,2(0,9)$ & 2,0 & 5,0 \\
\hline Ocorrência & $19(46,3)$ & $19(46,3)$ & $3(7,3)$ & $2,6(0,7)$ & 1,0 & 5,0 \\
\hline \multicolumn{7}{|l|}{ Lesões por pressão } \\
\hline Risco & $9(22,0)$ & $18(43,9)$ & $14(34,1)$ & $3,2(0,8)$ & 2,0 & 5,0 \\
\hline Ocorrência & $18(43,9)$ & $17(41,5)$ & $6(14,6)$ & $2,7(0,8)$ & 2,0 & 5,0 \\
\hline \multicolumn{7}{|l|}{ Erros de medicamentos } \\
\hline Risco & $20(48,8)$ & $11(26,8)$ & $10(24,4)$ & $2,9(1,1)$ & 2,0 & 5,0 \\
\hline Ocorrência & $31(75,6)$ & $8(19,5)$ & $2(4,9)$ & $2,3(0,7)$ & 1,0 & 5,0 \\
\hline \multicolumn{7}{|c|}{ Infecções relacionada à assistência à saúde } \\
\hline Risco & $11(26,8)$ & $17(41,5)$ & $13(31,7)$ & $2,8(0,9)$ & 1,0 & 5,0 \\
\hline Ocorrência & $11(26,8)$ & $24(58,5)$ & $6(14,6)$ & $2,7(0,8)$ & 1,0 & 5,0 \\
\hline
\end{tabular}

Tabela 2 - Análise por domínio de riscos e ocorrências de eventos adversos ( $\mathrm{n}=41$ ) e consistência interna

\begin{tabular}{|c|c|c|c|c|}
\hline Domínios & Média (DP*) & Mínimo & Máximo & Alfa de Cronbach \\
\hline Déficit de vigilância e julgamento clínico inadequado & $2,5(0,6)$ & 1,0 & 4,0 & 0,566 \\
\hline Déficit de defesa e delegação inapropriada & $2,0(0,8)$ & 1,0 & 4,0 & 0,778 \\
\hline Quedas & $2,9(0,7)$ & 1,5 & 5,0 & 0,648 \\
\hline Lesões por pressão & $2,9(0,7)$ & 2,0 & 5,0 & 0,630 \\
\hline Erros de medicamentos & $2,6(0,8)$ & 1,5 & 5,0 & 0,684 \\
\hline Infecção relacionada à assistência à saúde & $3,0(0,8)$ & 1,0 & 5,0 & 0,697 \\
\hline
\end{tabular}


Na Tabela 3, estão demonstradas as correlações de riscos e ocorrências de eventos adversos associadas às práticas assistenciais de enfermagem.

Observou-se correlação positiva entre eventos adversos relacionados a lesões por pressão e quedas $(p<0,001)$; erros de medicamentos e lesões por pressão ( $\mathrm{p}=0,004)$; e infecções relacionadas à assistência e erros de medicamentos $(\mathrm{p}=0,006)$.

Tabela 3 - Correlação entre riscos e ocorrências de eventos adversos na perspectiva de enfermeiros de um hospital de ensino $(n=41)$

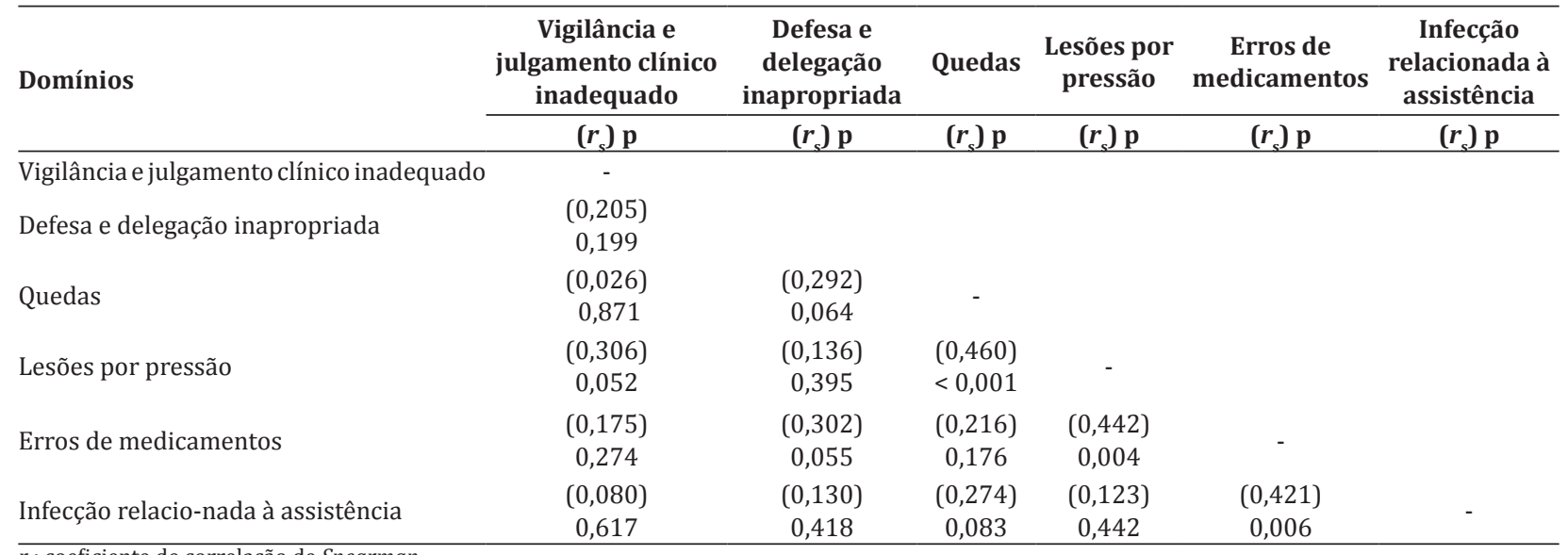

$r_{\mathrm{s}}$ : coeficiente de correlação de Spearman

\section{Discussão}

O estudo apresentou limitações quanto à aplicação em um único hospital e restrição da população na utilização do instrumento construído especificamente para enfermeiros. $\mathrm{Na}$ existência de uma equipe multiprofissional, os riscos e ocorrências de eventos adversos não podem ser atribuídos exclusivamente aos enfermeiros. No entanto, os resultados permitiram conhecer a perspectiva destes no que diz respeito à diferença na qualidade dos cuidados prestados e correlacionar os eventos adversos na segurança do paciente hospitalizado.

0 planejamento do cuidado seguro de enfermagem com ações voltadas para prevenção de eventos adversos deve ser baseado na vigilância direta ao paciente e no adequado julgamento clínico do enfermeiro, capaz de diagnosticar necessidades individuais de cada paciente. No cenário hospitalar, o despreparo de profissionais de saúde, devido à falta de conhecimento e visão crítica, pouca experiência e ausência de treinamentos, influencia no cuidado prestado e no processo de gerenciamento de risco das instituições de saúde ${ }^{(4-15)}$.
Na prática em saúde, o papel do enfermeiro na delegação de profissionais para a realização da assistência torna-se um ato de defesa do paciente, uma vez que ações voltadas para promoção da saúde e prevenção de riscos favorecem a segurança no cuidado prestado. 0 enfermeiro é um agente importante na delegação adequada de tarefas para alcançar os resultados esperados a partir do plano terapêutico estabelecido para o paciente ${ }^{(4-9)}$.

O Quality and Safety Education for Nurses Institute afirma a necessidade do profissional desenvolver conhecimento, habilidade e atitudes em torno de seis eixos considerados prioritários às competências para a segurança do paciente: cuidado centrado no paciente, trabalho em equipe e colaboração, prática baseada em evidência, melhoria da qualidade, segurança e informática $^{(10)}$.

Os achados apresentados apontaram correlação positiva entre a sobrecarga de trabalho dos enfermeiros e o risco de delegação inapropriada de profissionais menos preparados para realização dos cuidados. Infere-se, portanto, que a sobrecarga de trabalho influencia negativamente na tomada de decisão 
clínica durante a gestão do cuidado, o que pode favorecer a ocorrência de incidentes. Esse achado corrobora com estudo realizado em hospitais Finlandeses, cujo excesso de trabalho atribuídos aos profissionais da enfermagem aumentou o risco de incidentes de segurança e eventos adversos entre 8 e $34,0 \%$ com chance de um paciente morrer em $40,0 \%{ }^{(11)}$.

Ao analisar o domínio relacionado a quedas de pacientes, grande parte dos enfermeiros $(46,3 \%)$ afirmou que algumas vezes esses eventos acontecem na instituição, o que necessita ser cautelosamente investigado pelo potencial de causar danos e aumentar o tempo de internação. Estudo desenvolvido em ambiente hospitalar sustenta que quedas ainda continuam ocorrendo e causando consequências graves aos pacientes, estimando taxa de 12,6 para cada 1.000 pacientes por $\mathrm{dia}^{(12)}$.

Os resultados relacionados a quedas apontaram correlação positiva com outros tipos de eventos adversos como as lesões por pressão. Esse achado chama a atenção para a necessidade de compreender a complexidade do ambiente onde acontece o cuidado e a relação entre os diversos riscos e falhas da assistência. Ressalta-se, portanto, que a assistência de enfermagem não pode ser pautada em um processo fragmentado e a visão holística do cuidado tem por finalidade detectar em tempo oportuno eventuais alterações do estado clínico do paciente que, se não considerados, podem ampliar o risco de eventos adversos em todo processo assistencial ${ }^{(13)}$.

Quanto à ocorrência de lesão por pressão, apesar dos esforços contínuos para prevenção como a execução de medidas para análise dos riscos e o desenvolvimento de protocolos para melhoria da qualidade da assistência ${ }^{(14)}$ ainda se observa sua recorrência nas instituições de saúde. Nesse estudo, para 14,6\% dos enfermeiros sempre ocorre lesões por pressão. Estudo transversal realizado em hospital universitário apontou dados ainda maiores, com prevalência de lesões por pressão em $40,0 \%{ }^{(14)}$.

Evidenciou-se, ainda, que eventos adversos relacionados às lesões por pressão foram menos as- sociados aos profissionais que participaram de treinamento em segurança do paciente. Esta associação infere que aqueles profissionais com maiores esclarecimentos sobre a temática possuem uma visão mais crítica do ambiente de trabalho e maior percepção de controle dos riscos assistenciais, conferindo maior segurança ao processo de trabalho.

Enfermeiros com maior capacidade de autorreflexão e discernimento apresentam pensamento mais crítico em relação à prática clínica e a qualificação dos enfermeiros influencia de forma significativa o desenvolvimento desse pensamento crítico $^{(15)}$. Essa realidade corrobora com estudo que mostra que ações de educação continuada voltadas para redução de riscos e ocorrências de eventos adversos evitáveis favorecem o aumento da confiabilidade do sistema e dos processos de trabalho na enfermagem ${ }^{(16)}$.

Dos domínios explorados nesse estudo, erros de medicamentos apontou entre os resultados com menor incidência. Apenas 4,9\% dos enfermeiros consideraram a ocorrência desses eventos na instituição. Estudo de comparação realizado em hospitais Portugueses apontou resultados ainda menores, com afirmação de $0,9 \%$ dos enfermeiros para eventos adversos com medicamentos ${ }^{(6)}$. Esses achados são relevantes e podem estar associados com a implantação das metas internacionais de segurança do paciente, por meio do gerenciamento de riscos, com a finalidade de reduzir danos evitáveis no processo que envolve a terapia medicamentosa ${ }^{(17)}$.

Os erros de medicamentos foram associados com outros tipos de eventos como infecções e lesões por pressão, mais uma vez apontando para os multifatores envolvidos na ocorrência de um incidente. Erros no processo que envolve a terapia medicamentosa como a ausência da higienização das mãos antes do preparo e administração das medicações podem potencializar o surgimento de outros tipos de eventos adversos na assistência como as infecções ${ }^{(6)}$. Quando envolvem eventos como lesões por pressão em pacientes hospitalizados podem estar associados pela diminuição da percepção sensorial devido ao excesso 
de sedativos, analgésicos e relaxantes musculares ${ }^{(18)}$.

Os riscos e ocorrências das infecções associadas às práticas assistenciais foram os mais frequentes, pois apenas $26,8 \%$ dos enfermeiros negaram a ocorrência desses eventos na instituição. Estudo realizado em Portugal também identificou maior frequência desse tipo de evento, evidenciando que apenas 30,5\% dos enfermeiros negaram sua ocorrência ${ }^{(6)}$.

As infecções associadas às práticas assistenciais são um dos principais desafios para a manutenção da qualidade da assistência. Trata-se de um problema de saúde pública que gera impacto social e financeiro, o qual poderia ser investido no controle e prevenção de infecções, com a implementação de normas, diretrizes e indicadores. Contudo, para o alcance de resultados positivos na assistência, há necessidade de oferecer recursos materiais, humanos e melhores condições organizacionais ${ }^{(19)}$.

O incentivo atual para a prevenção e redução de eventos adversos é a adoção de estratégias multifacetadas, a fim de trabalhar o máximo de fatores causais envolvidos na ocorrência dos incidentes ${ }^{(1-3)}$. A estimulação do raciocínio clínico e reflexivo é fundamental no desempenho de habilidades e capacidades imprescindíveis do enfermeiro(13-15), as quais são componentes essenciais a serem incorporados à cultura de segurança. Para isso, a educação permanente e continuada deve ser inserida como ferramenta no processo de trabalho para promoção de mudanças no comportamento e postura profissional, determinantes na tomada de decisões assistenciais ${ }^{(16-20)}$.

\section{Conclusão}

A análise evidenciou que a ocorrência de eventos adversos nos cuidados assistenciais como lesões por pressão e infecções relacionada a assistência à saúde foram as mais frequentes na percepção de enfermeiros. Dos domínios explorados, erros de medicamentos apontou entre os resultados com menor incidência. A ocorrência desses eventos mostrou ligação para o surgimento de outros tipos de eventos adversos durante o processo assistencial de enfermagem, como a associação de erros de medicamentos com infecções e lesões por pressão. Por outro lado, verificou-se também associação significante entre o menor risco de ocorrência de lesões por pressão para enfermeiros que realizaram curso em segurança do paciente.

\section{Colaborações}

Amaral RT e Bezerra ALQ contribuíram na concepção do projeto, análise e interpretação dos dados, redação do artigo e revisão crítica relevante do conteúdo intelectual. Teixeira CC, Paranaguá TTB, Afonso TC e Souza ACS colaboraram com análise e interpretação dos dados, redação do artigo e revisão crítica relevante do conteúdo intelectual e aprovação da versão final a ser publicada.

\section{Referências}

1. D'Amour D, Dubois CA, Tchouaket E, Clarke S, Blais R. The occurrence of adverse events potentially attributable to nursing care in medical units: cross sectional record review. Int J Nurs Stud. 2014; 51(6):882-91. doi: http://dx.doi.org/10.1016/j. ijnurstu.2013.10.017

2. Sousa P, Uva AS, Serranheira F, Uva MS, Nunes C. Patient and hospital characteristics that influence incidence of adverse events in acute public hospitals in Portugal: a retrospective cohort study. Int J Qual Health Care. 2018; 30(2):132-7. doi: http://dx.doi.org/10.1093/intqhc/mzx190

3. Duarte SCM, Stipp MAC, Silva MM, Oliveira FT. Adverse events and safety in nursing care. Rev Bras Enferm. 2015; 68(1):136-46. doi: http:// dx.doi.org/10.1590/0034-7167.2015680120p

4. Françolin L, Gabriel CS, Bernardes A, Silva AEBC, Brito MFP, Machado JP. Patient safety management from the perspective of nurses. Rev Esc Enferm USP. 2015; 49(2):277-83. doi: http://dx.doi. org/10.1590/S0080-623420150000200013

5. Siman AG, Cunha SGS, Brito MJM. Nursing actions for patient safety in hospitals: integrative review. Rev Enferm UFPE on line [Internet]. 2017 [cited Feb 17, 2019];11(Suppl. 2):1016-24. Available 
from:https://periodicos.ufpe.br/revistas/ revistaenfermagem/article/view/13472/16174

6. Castilho AFOM, Parreira PMD, Martins MMFPS. Nursing Care and Adverse Events at inpatients in acute hospitals: the root cause analysis. Riase online. 2016; 2(2):605-23. doi: http://dx.doi. org/10.24902/r.riase.2016.2(2).600

7. Neves T, Rodrigues V, Graveto J, Parreira P. Scale of adverse events associated to nursing practices: a psychometric study in Portuguese hospital contexto. Rev Latino-Am Enfermagem. 2018; 26:e3093. doi: http://dx.doi.org/10.1590/15188345.2595 .3093

8. Araujo MAN, Lunardi Filho WD, Silveira RS, Souza JC, Barlem ELD, Teixeira NS. Segurança do paciente na visão de enfermeiros: uma questão multiprofissional. Enferm Foco [Internet]. 2017 [citado 2019 fev 07];8(1):52-6. Disponível em: http:// revista.cofen.gov.br/index.php/enfermagem/article/view/984

9. Tomaschewski-Barlem JG, Lunardi VL, Barlem ELD, Ramos AM, Silveira RS, Vargas MAO. How have nurses practiced patient advocacy in the hospital context? - a foucaultian perspective. Texto Contexto Enferm. 2016; 25(1):e2560014. doi: dx.doi. org/10.1590/0104-0707201600002560014

10. Quality and Safety Education for Nurses (Qsen) Institute. Qsen competencies [Internet]. 2019 [cited Feb 17, 2019]. Available from: http://qsen. org/competencies/pre-licensure-ksas/

11. Fagerström L, Kinnunen M, Saarela J. Nursing workload, patient safety incidents and mortality: an observational study from Finland. BMJ Open. 2018; 8:e016367. doi: http://dx.doi.org/10.1136/ bmjopen-2017-016367

12. Abreu HCA, Reiners AAO, Azevedo RCS, Silva AMC, Abreu DROM, Oliveira AD. Incidence and predicting factors of falls of older inpatients. Rev Saúde Pública. 2015; 49(37):1-8. doi: http://dx.doi. org/10.1590/S0034-8910.2015049005549
13. Savieto RM, Leão ER. Nursing assistance and Jean Watson: a reflection on empathy. Esc Anna Nery. 2016; 20(1):198-02. doi: http://dx.doi. org/10.5935/1414-8145.20160026

14. Bernardes RM, Caliri MHL. Pressure ulcer pre valence in emergency hospitals: a cross-sectional study. Online Braz J Nurs [Internet]. 2016 [cited Aug 24, 2019]; 15(2):236-44. Available from: http://www.objnursing.uff.br/index.php/nursing/article/view/5391

15. Fen-Fang C, Shu-Yueh C, Hsiang-Chu P. Self-reflection and critical thinking: the influence of professional qualifications on registered nurses. Contempt Nurse. 2019; 55(1):59-70. doi: http://dx. doi.org/10.1080/10376178.2019.1590154

16. Flores GE, Oliveira DLL, Zocche DAA. Educação permanente no contexto hospitalar: a experiência que ressignifica o cuidado em enfermagem. Trab Educ Saúde. 2016; 14(2):487-04. doi: http:// dx.doi.org/10.1590/1981-7746-sip00118

17. World Health Organization. Medication Without Harm - Global Patient Safety Challenge on Medication Safety. Geneva: World Health Organization; 2017.

18. Pachá HHP, Faria JIL, Oliveira KA, Beccaria LM. Pressure ulcer in Intensive Care Units: a case-control study. Rev Bras Enferm. 2018; 71(6):3027-34. doi: dx.doi.org/10.1590/0034-7167-2017-0950

19. Oliveira HM, Silva CPR, Lacerda RA. Policies for control and prevention of infections related to healthcare assistance in Brazil: a conceptual analysis. Rev Esc Enferm USP. 2016; 50(3):505-11. doi: dx. doi.org/10.1590/S0080-623420160000400018

20. Mello JF, Barbosa SFF. Patient safety culture in an intensive care unit: the perspective of the nursing team. Rev Eletr Enf. 2017; 19:a07. doi: http:// dx.doi.org/10.5216/ree.v19.38760 